\title{
Discrimination of restrictions in sequentially blocked auditory displays: Shifting block designs*
}

\author{
IRWIN POLLACK† \\ University of Michigan, Ann Arbor, Michigan 48104
}

Finite-state Markov sequences were generated by a digital computer, translated to electrical pulse trains, and converted to sound by earphones. The sequences were constrained in terms of the maximum number of occurrences of any state within any block of successive items. The discriminability among such sequences was measured in terms of the threshold interstate interval difference that is required to achieve a given level of discrimination among the sequences. These thresholds are closely related to the number of selections available to the source generator for each position of the sequence.

This study represents one of a continuing series of attempts to explore the sensitivity of $O s$ to sequential restrictions imposed on finite-state statistical generators. The distinctive feature of the experimental strategy is to convert the output of the statistical generators to sound. The strategy takes advantage of the high display rates possible with auditory signals and of the specialized information-processing capability of the auditory system for the analysis of sequentially encoded information (Pollack. 1968a, b, 1969).

The specific form of sequential restriction examined in the present report is the maximum number of occurrences of any state within any defined set of consecutive items of the sequence. The test has been labeled "shifting block design." Sequential restrictions are imposed upon a defined set of consecutive itenis, or a block, which is shifted along the entire sequence. The procedure differs from those that might arbitrarily divide the sequence into successive nonoverlapping blocks. The essential notion under test is that discriminability is related to the number of choices available to the statistical generator for each position of the sequence. Or, more specifically, discriminability between two sequential constraints is related to the ratio of the number of choices available to the statistical source under each of the constraints.

*This research was supported by Grant GB 6148 from the National Science Foundation. The author is indebted to K. Weidner, who supervised the experimental tests; to L. Radin. who wrote the PDP-8 computer program for the experimental and simulation tests; and to R. Hsieh, who wrote the data-processing program.

$\dagger$ Mailing address: Mental Health Research Institute, University of Michigan, Ann Arbor. Michigan 48014. sequence, because missing states become more eligible for selection than more frequent states.

Figures 1 and 2 present the results of a program simulation of SBDI, in which 200,000 tabulations were made of the number of selections available for each sequence position. Figure 1 examines the average number of selections available to binary sequences at each position of the sequence. The parameter is the maximum number of occurrences of either binary element, expressed relative to the block length. With the most severe restriction, $\mathrm{m} \cdot \mathrm{R}-\mathrm{B}=0$, only a single selection is possible with only two elements. The most severe restriction but one, $m \cdot R-B=1$, produces a large change in the proportion of selections available. Successively weaker restrictions produce smaller proportional changes in the number of selections. Greater relative limitations are imposed upon longer sequences for a given restriction, when expressed in the manner of Fig. 1. For example, consider the restriction $m \cdot R-B=2$. For $m=2$, $R=3, B=4$, only two of the eight possible preceding triplets limit the subsequent selection to a single choice; for $m=2$, $\mathrm{R}=4, \mathrm{~B}=6,10$ of the 32 possible preceding 5-tuples limit the subsequent selection to a single choice; and, with the restriction $m=2, \quad R=501, \quad B=1,000$, nearly half of sequences of Length 999 limit the subsequent selection to a single choice. The points of Fig. 1 are coded in terms of the observed proportion of terminated trials (to be defined) when a given condition is paired against unrestricted random sampling of the two states. More difficult discrimination is associated with a greater proportion of terminated trials, which is reflected in terms of a greater filling of the points. In terms of this rough measure of discrimination, poorer discrimination, relative to the unrestricted condition, is associated with conditions with a greater average number of choices per position.

Figure 2 extends the analysis to

Table 1

Illustration of Shifting Block Design Restrictions

$$
\text { SHIFIINC, BLOCK OESIGN }
$$
Design I, or SBDI, all remaining eligible states are equally likely. For example, in the sequence ...100 with $R=3$, a random selection is made between States 0 and 1. In Shifting Block Design II, or SBDII, all remaining possibilities were equally likely. For the same sequence, $\ldots 100$ with $\mathrm{R}=3$, a random selection is made among two $1 \mathrm{~s}$ and one 0 , such that the probability of selecting 1 is twice that of selecting 0 . The second rule tends to even out localized perturbations in the

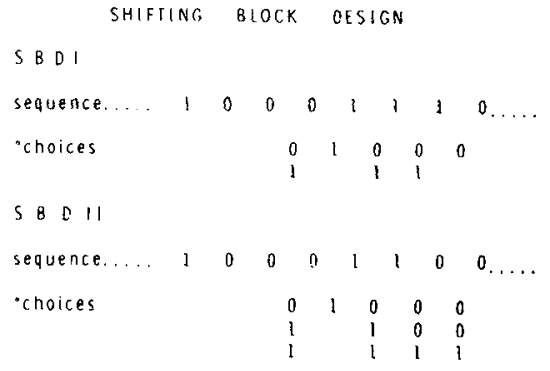

$\cdot m=2,8=4, R=3$ 


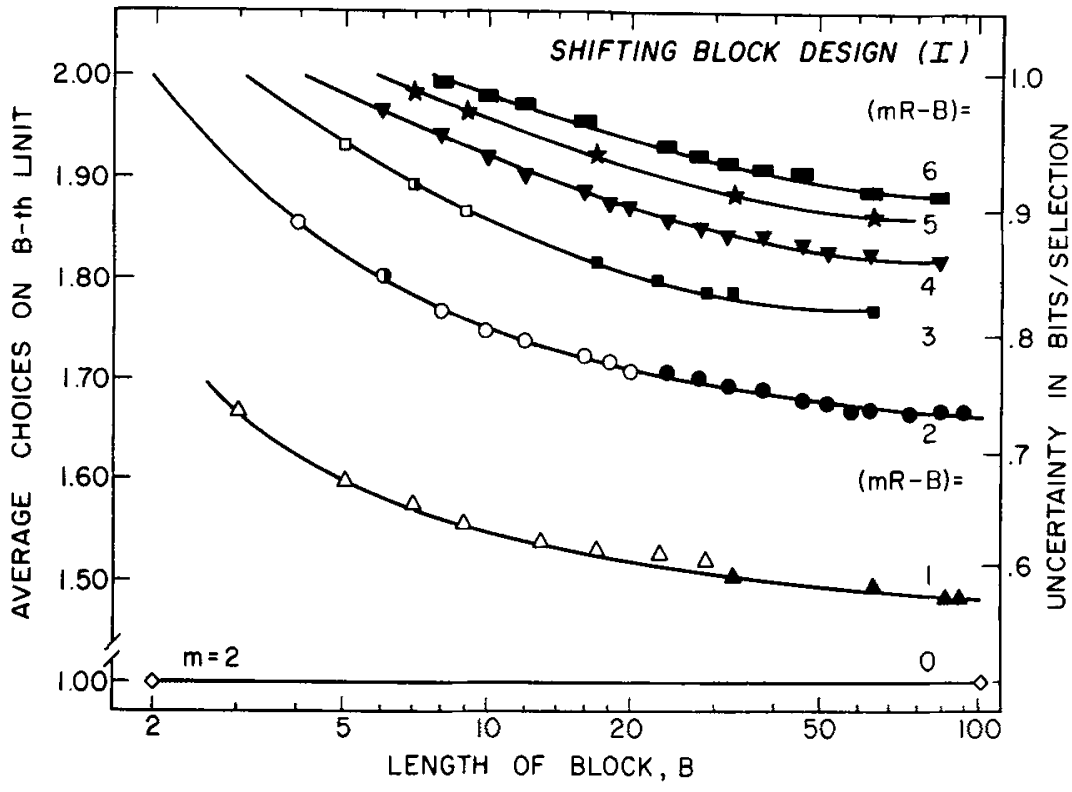

Fig. 1. Effect of shifting block restrictions upon the number of selections available for the two-state sequences. The parameter of Figs. 1 and 2 is the maximum number of occurrences within a consecutive block of items of either binary state, relative to the length of the block. The ordinate is broken to accommodate periodic sequences, $m \cdot R-B=0$. The right ordinate represents the equivalent uncertainty in bits per selection. The degree of filling of the points reflects the proportion of trials that exceeded the capacity of the program (see text). Procedure: Shifting Block Design I for all figures.

multistate sequences where $m \geqslant 2$. The abscissa is the number of possible states. The three sections of Fig. 2 represent three block lengths, $B$. The parameter is the restriction imposed with respect to the maximum number of occurrences of each state within the block. The dashed reference line at $45 \mathrm{deg}$ represents the maximum number of possible selections with the indicated number of states. The scale is logarithmic, so that equal vertical extents represent equal changes in informational uncertainty. The thin dotted lines connect points with an equal $m \cdot R$ product. With respect to the dashed line representing no restrictions, there is a relative advantage in favor of the largest number of states. For example, in the left graph, relative to the maximum number of 2,3 , and 6 possible selections, about 1.9 , 2.3 , and 3.0 choices are available, respectively, under the $m \cdot R$ product of 6 . As in Fig. 1, the points are coded in Fig. 2 to reflect the observed proportion of terminated trials when a given condition is paired against the unrestricted condition. In terms of the rough measure of discrimination, poorer discrimination is again associated with smaller differences in uncertainty.

The notion under experimental test is that an empirical measure of interval discriminability among the sequences will reflect the relative number of choices available to the sequence generator. More into interval-coded electrical pulse trains and converted into sound by earphones (Koss PRO-4). For example, the binary sequence $\mathrm{ABA} \ldots$ is represented by: a pulse, an interpulse interval $\mathrm{IPI}_{A}$, a pulse, interpulse interval $\mathrm{IPI}_{B}$, a pulse, interpulse interval $\mathrm{IPI}_{\mathrm{A}}$, a pulse,.... With a mean interpulse interval, $\mathrm{IPI}_{\mathrm{c}}$, of $0.5 \mathrm{msec}, 2,000$ states per second were presented to the listener.

The sequences were defined in terms of: $\mathrm{N}$, the number of interpulse intervals in the entire sequence; $m$, the number of different states available to the sequence; $R$, the maximum number of occurrences of each state within a block, $B$, of consecutive items; $\mathbf{I P I}_{C}$, the average interpulse interval; and DIPI, the difference in the interpulse interval between adjacent states.

The listener heard four pulse trains. Three pulse trains employed Restriction $R_{S}$, the smaller of two restrictions over the block of $B$ intervals; one pulse train employed Restriction $\mathbf{R}_{\mathbf{L}}$, the larger of the two restrictions over the same block length. The listener's task was to indicate which one of the four pulse trains sounded different from the other three.

An adaptive stimulus programming procedure of variable step size (Taylor \& Creelman, 1967) manipulated DIPI, the difference between adjacent states. When the listener selected the wrong pulse train of 4, DIPI was increased; when he selected the correct pulse train, DIPI was decreased. Thus, the procedure aimed to converge upon $50 \%$ correct in a four-interval forced-choice test.

When DIPI is zero, all sequences consist of repeated occurrences of a single state

\section{Procedure}

Finite-state sequences were translated

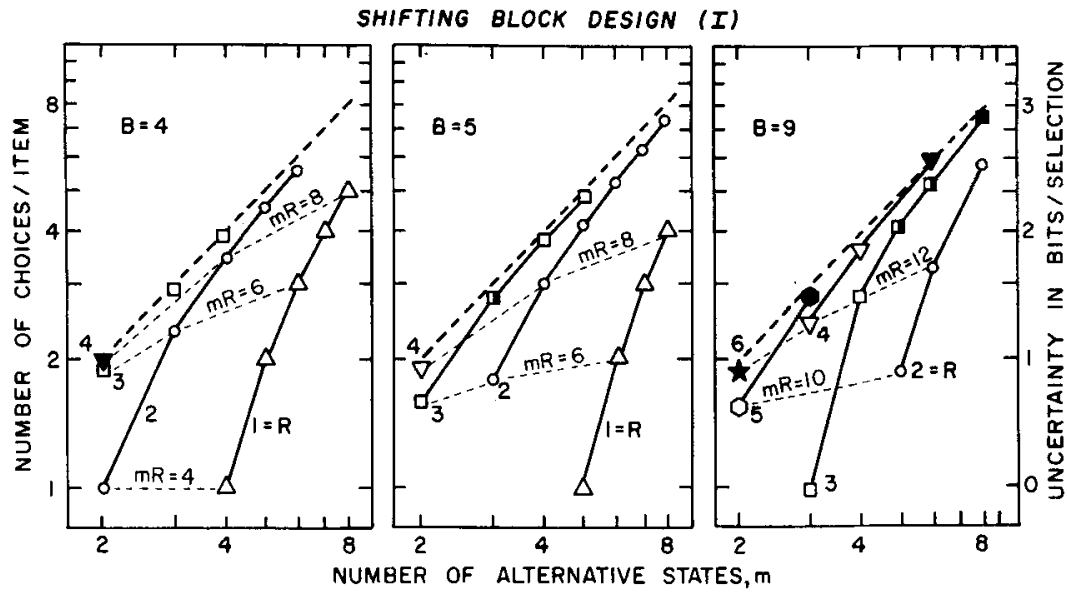

Fig. 2. Effect of shifting block restrictions upon the number of selections available for multistate sequences. The left ordinate seale is the average number of equally likely choices available for selection. The right ordinate represents the equivalent uncertainty in bits per selection. The dashed reference line represents the available number of choices for unrestricted messages. The three sections represent three different block lengths. The thin dashed lines connect points of an equal $m \cdot R$ product. 


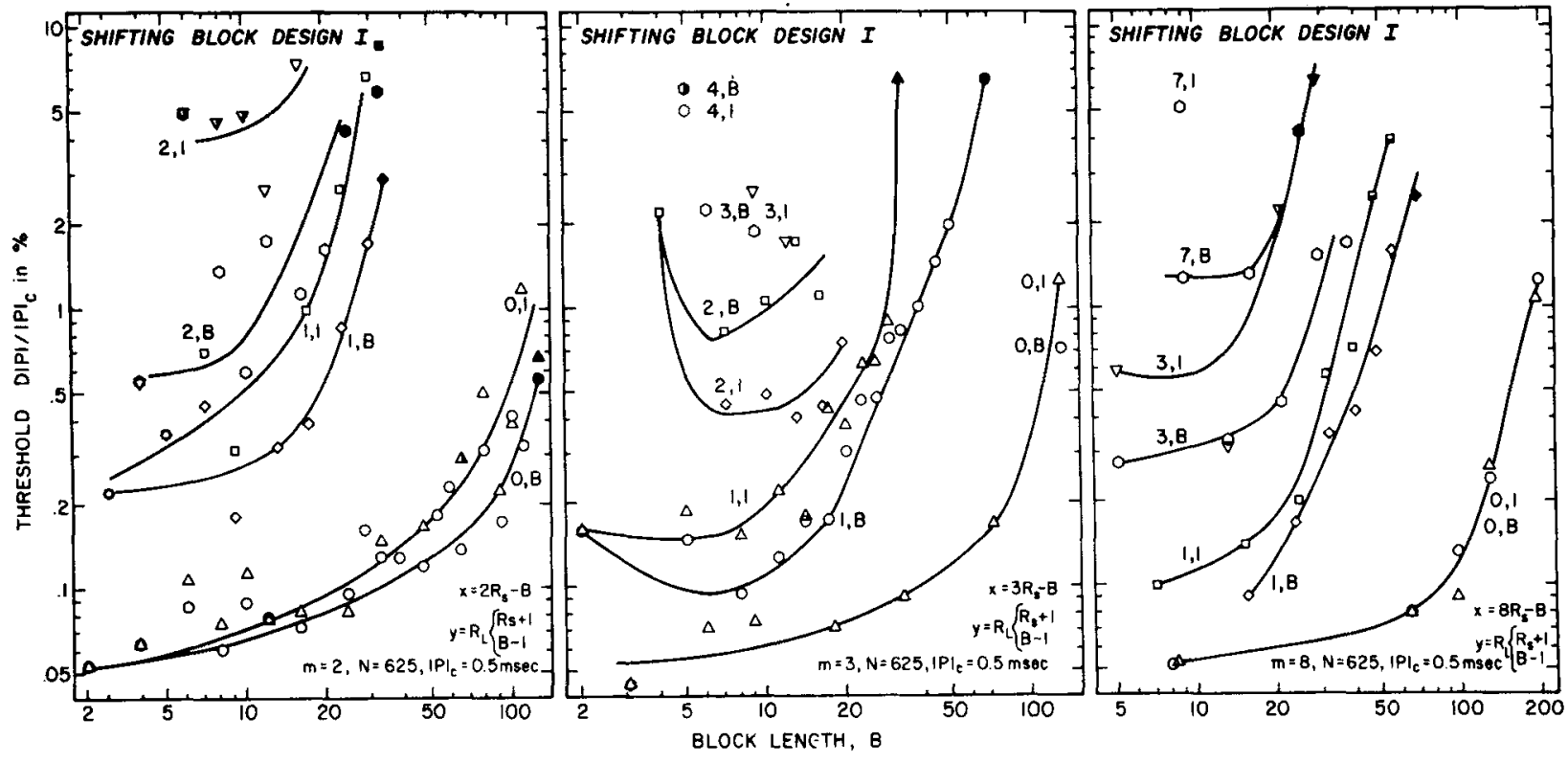

Fig. 3. Discrimination thresholds associated with binary (left section), trinary (middle section), and octal-encoded (right section) sequences under shifting block restrictions. The parameter represents both $S$ and $L$, expressed relative to $B$, as shown in the lower right corner. Specifically, in the left section, 0,1 implies $2 R_{S}-B=0$ and that $R_{L}=R_{S}+1$. Similarly, in the left section, 2 , $B$ implies that $2 R_{S}-B=2$ and that $R_{L}=B-1$.

It was assumed that threshold DIPI would reflect the relative discriminability of sequential constraints. If DIPI were unrelated to the discrimination of sequential restrictions, the adaptive programming procedure would not converge upon a stable threshold. Since the procedure converged upon stable DIPI thresholds, it is assumed that DIPI thresholds provide an indirect measure of discriminability among the sequences. Independent tests show nearly equivalent results by modification of sequential restrictions and fixing DIPI, and by modification of DIPI and fixing sequential restrictions (Pollack, 1968a).

The Os had extersive previous experience listening to auditory pulse trains: 1-6 semesters, $5 \mathrm{~h}$ per week. Each point represents the average of at least two thresholds by each of 15 listeners. Under unfavorable sequential restrictions, the adaptive procedure may have sought a value for DIPI which exceeded the capability of the program. The limit was $0.737 \mathrm{msec} /(\mathrm{m}-1)$ or, at an $\mathrm{IPI}_{\mathrm{c}}=0.5 \mathrm{msec}$, the limiting DIPI was $147 \% /(\mathrm{m}-1)$. When the sought value exceeded the limiting condition, an observation was provided at the limiting condition. If an incorrect response was then made, the trial was terminated. If a correct response was made at the limiting condition, DIPI was modified and testing was continued. Terminated trials were not repeated. The following code is employed to indicate the proportion of terminated trials: open points, $<10 \%$ terminated trials; half-filled points, $10 \%-20 \%$ terminated trials; filled points, $>20 \%$ terminated trials. Geometric means, excluding the terminated trials, were employed with $<10 \%$ terminated trials; medians, including the terminated trials, were employed with $\geqslant 10 \%$ terminated trials. Each listener completed 396 experimental conditions under SBDI and 198 experimental conditions under SBDII.

\section{RESULTS}

\section{Comparison Between SBDI and SBDII}

Two comparisons were made between interstate interval thresholds, DIPI, under SBDI and SBDII. For each of 97 experimental conditions under SBDI and II, the following measures were obtained: geometric mean DIPI thresholds, excluding terminated trials; median DIPI thresholds, including terminated trials; and the proportion of terminated trials. The correlation coefficients for corresponding experimental conditions (and the corresponding averages for SBDI and SBDII) were $0.93(2.0 \%$ and $2.6 \%$ DIPI); $0.76(3.6 \%$ and $6.4 \% \mathrm{DIPI})$; and $0.90(7.7 \%$ and $10 \%$ terminated trials, respectively. The analysis was extended to another set of 79 conditions. The correlation coefficients were: $0.89(1.8 \%$ and $2.2 \%$ DIPI); 0.78 (9.0\% and $12 \%$ DIP1; and 0.96 ( $23 \%$ and $28 \%$ terminated trials), respectively. The general picture, then, is that SBDII results in somewhat higher thresholds and in a somewhat higher proportion of terminated trials than does SBDI, but the overall correlation between the two methods is high. Higher thresholds are reasonable if discriminability is related to the degree of sequential constraint imposed by the restrictions. In SBDII the more frequently occurring states within any block become less eligible for selection. The net effect is to reduce the likelihood of eliminating any state within SBDII.

\section{Binary Sequences}

The left section of Fig. 3 presents interstate thresholds associated with binary-coded sequences, $m=2$, for SBDI. The parameter on the curves is the $R_{S}, R_{L}$ combination for each block length, expressed in the manner shown in the lower right corner of the left section of Fig. 3.

The lower set of curves represents experimental conditions in which the more severe restriction resulted in strictly periodic sequences, i.e., $2 R_{S}-B=0$. Such sequences were pitted against only slightly less-restricted sequences, $R_{L}=R_{S}+1$, and against nearly unrestricted sequences, $R_{L}=(B-1)$. The failure to employ $R_{L}=B$ for the other extreme was inadvertent, not deliberate. Cousistent thresholds are obtained for block lengths of over 100. The effect of $R_{L}$ is relatively small. For comparison, inspection of Fig. 2 shows that the major jump in the number of possible selections is from a strictly periodic sequence (one choice per item) to the next less severe, i.e., larger, restriction. Additional restrictions are associated with proportionately lower changes in uncertainty. 


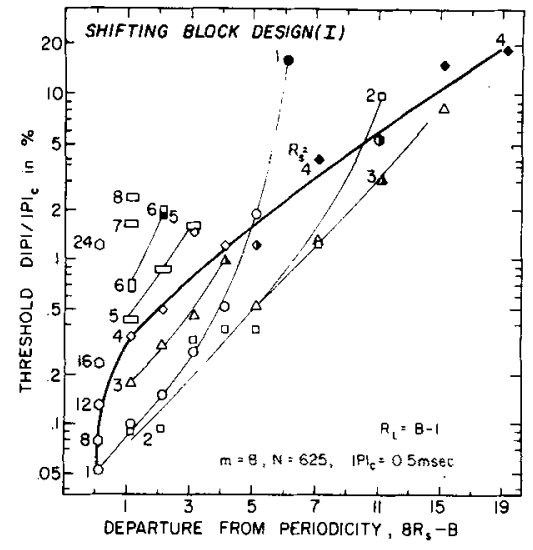

Fig. 4. Discrimination thresholds associated with octal-encoded sequences. The abscissa is the departure of $R_{S}$ from strict periodicity. The parameter is $R_{S}$ Thin lines connect points of equal $R_{S}$. Note the change in scale beyond 7 on the abscissa. The heavy curve is drawn through points for $R_{S}=4$.

The middle set of curves of the left section of Fig. 3 represent the smallest departure from perfect periodicity, $2 \mathrm{R}_{\mathrm{S}}-\mathrm{B}=1$. Again, two extremes are presented for $R_{L}$, where $R_{L}=R_{S}+1$ and $\mathrm{R}_{\mathrm{L}}=(\mathrm{B}-1)$. Substantially larger DIPI thresholds are required with the slight relaxation in $\mathrm{R}_{S}$. Consistent thresholds are achieved for block lengths of only about 28. For comparison, inspection of Fig. 2 reveals that a smaller potential change in uncertainty, relative to the unrestricted case, is associated with $2 R_{S}-B=1$ than with $2 R_{S}-B=0$. Compare, for example, the distances to the dashed reference line in Fig. 2 between $m=2, R=2, B=4$ (left section with $m \cdot R=B$ ) and $m=2, R=3$, $\mathrm{B}=5$ (middle section with $\mathrm{m} \cdot \mathrm{R}=\mathrm{B}+1$ ).

In addition, relative to the lowest set of curves, differences in $R_{L}$ take on added significance for DIPI threshoids. Other intermediate levels of $R_{L}$ (not plotted) tend to fall between the extremes of $R_{L}$. Comparison with Fig. 2 suggests greater changes in relative uncertainty associated with changes in $R_{L}$, when $R_{S}$ departs from the strictly periodic condition, $\mathrm{m} \cdot \mathrm{R}-\mathrm{B}=0$.

The upper curves of the left section of Fig. 3 represent $2 R_{S}-B=2$. Consistent discrimination is achieved only for $\mathrm{R}_{\mathrm{L}}=(\mathrm{B}-1)$ for block lengths to only about 15. Consistent discrimination is not achieved for $R_{L}=R_{S}+1$. For comparison, inspection of Fig. 2 reveals that the maximum available difference in uncertainty is small for $2 R_{S}-B=2$, as with $\mathrm{R}=3$ in the left section for $m=2$.

In several comparisons, therefore, the discriminability thresholds of Fig. 3 tend to be quantitatively reflected in the uncertainty analysis of Fig. 2 .

\section{Multistate Sequences}

The middle section of Fig. 3 presents a corresponding analysis for three-state sequences, $m=3$. Most of the features of the left section are also reflected within the middle section. From Fig. 2 we note that a given $(m \cdot R-B)$ difference-shown by thin dashed lines-produces relatively larger differences in uncertainty as the number of states is increased. For example, in the middle section of Fig. 2, the thin line connecting the pairs, $\mathrm{m}=2, \mathrm{R}=3 ; \mathrm{m}=3$, $R=2 ;$ and $m=6, R=1$, marks conditions where $m \cdot R=6$. Relative to the maximum number of choices indicated by the dashed reference line, uncertainty differences grow with $m$ for a fixed $m \cdot R$ product for a constant block length. This is reflected, for example, in the lower DIPI thresholds associated with $m \cdot R_{S}-B=1$ in the middle section than in the left section of Fig. 3. The upper limit for discrimination is still apparently obtained at about $\mathrm{R}_{\mathrm{S}}=1+\mathrm{B} / \mathrm{m}$, or: $(\mathrm{B}+\mathrm{m}) / \mathrm{m}$.

The right section of Fig. 3 presents a corresponding analysis for octal-encoded sequences, $m=8$. The major trends suggested for $m=2$ (left section) and $\mathrm{m}=3$ (middle section) are also reflected for $m=8$ (right section). DIPI thresholds rise as the restrictions are successively relaxed. The upper limit of discrimination remains in the ballpark of $R=1+B / m$, or $(B+m) / m$.

Figure 4 considers the results of octal-coded sequences, $m=8$, in more detail. The abscissa is the departure from periodicity, expressed relative to the block length. The parameter is $R_{S}$. Thus, the quantity $8 R_{S}-B=0$, for example, obtains for the following combinations of $\mathrm{R}_{\mathrm{S}}$ and $\mathrm{B}: 1,8 ; 8,64 ; 12,96 ; 16,128$; and 24,192 . The heavy curve is drawn through $\mathrm{R}_{\mathrm{S}}=4$. The main result is a $2 \frac{1}{2}$-decade change in thresholds with greater and greater departures from periodicity. Figure 4 also reflects the general rise in thresholds with increasing block length, as found in the three sections of Fig. 3. The relationship is not always monotonic, e.g., with a departure of 5 , lowest thresholds are obtained with $\mathrm{R}_{\mathrm{S}}=2$, and highest thresholds are obtained with $\mathrm{R}_{\mathrm{S}}=1$. $\mathrm{A}$ related anomoly is also revealed by the conditions represented by several functions in the middle section of Fig. 3. The anomoly is probably related to the use of $\mathrm{R}_{\mathrm{L}}=\mathrm{B}-1$ rather than to the extreme $R_{L}=B$

\section{DISCUSSION}

A strong quantitative statement of the relationship between discriminability thresholds and shifting block constraints is suggested by the results: For a given block length, discriminability thresholds are monotonically related to the difference in the informational uncertainty of selection available to the statistical generator.

For a given set of restrictions, expressed in terms of departure from a strictly constrained sequence, differences in uncertainty decrease slightly with longer block lengths. Discriminability thresholds, on the other hand, may increase sharply with longer block lengths. One implication is that uncertainty alone is not the entire story. The quality of the running sequential analysis, implied by the shifting block restrictions, becomes less and less effective as the block length is increased. The interaction with block length becomes progressively more severe as the sequences become progressively less constrained, as shown in Fig. 3.

It is misleading to suggest, even by omission, that the auditory system is making the running uncertainty analysis implied by the results. More likely, the auditory system is providing a running temporal or spectrum analysis upon the interval-coded messages, where uncertainty is roughly related to the width of the spectral distribution. The spectrum is best defined for periodic signals and is progressively more poorly defined as the signals depart from periodicity. In these terms, the limitations upon block length are presumably related to limitations upon the temporal or spectral resolution of the auditory system.

\section{REFERENCES}

POLLACK, I. Periodicity discrimination for auditory pulse trains. Journal of the A :oustical Society of America, 1968a, 43, 1113-1119.

POLLACK, I. Discrimination of changes in Markov sequences with the first $\mathrm{N}$-th orders statistically independent. Proceedings of the 76 th Annual Convention, APA, 1968b. 125-126.

POLLACK, I, Depth of sequential auditory information processing. Journal of the Acoustical Society of America, 1969, 46, 952-964.

TAYLOR, M. M., \& CREELMAN. C. D. PEST: Efficient estimates on probability functions. Journal of the Acoustical Society of America, $1967.41,782-787$.

(Accepted for publication August 4, 1970.) 